\title{
Convergence of Alternating Domain Decomposition Schemes for Kinetic and Aerodynamic Equations
}

\author{
by AXEL KLAR
}

\begin{abstract}
A domain decomposition scheme linking linearized kinetic and aerodynamic equations is considered. Convergence of the alternating scheme is shown. Moreover the physical correctness of the obtained coupled solutions is discussed.
\end{abstract}

\section{Introduction}

Domain decomposition methods date back until 1869 when Schwarz proved the convergence of his alternating method for elliptic equations [12]. A systematic investigation of the convergence properties of this method was done by Lions in a series of papers [10] for various linear and nonlinear elliptic equations.

Recently domain decomposition problems involving kinetic and aerodynamic equations have become of considerable interest e.g. in space flight problems. According to different heights of the space vehicle during the reentry different equations have to be solved in different subdomains of the computational domain.

The kinetic equation involved here is usually the Boltzmann equation. It must be solved in low density regions and inside of shock- and kinetic-boundary-layers. In high density regions outside of shock and boundary layers the aerodynamic equation should be used. This is mainly due to the large computation times needed for the numerical solution of the Boltzmann equation if the mean free path is small, i.e. in high density regions. Apart from the determination of the subdomains which allow an aerodynamic modelling and of those where a Boltzmann simulation is required, there is one major problem: The coupling of the solution procedures for the kinetic equation on the one and the aerodynamic equation on the other subdomain.

When the coupling conditions are defined the domain decomposition problem is usually solved by an iterative procedure solving in turn the kinetic and aerodynamic equations in their respective subdomains. This is a generalization of the above mentioned Schwarzmethod. We roughly sketch the scheme which is up to now the only one being used in numerical calculations, see e.g. Bourgat et al. [3] or Lukschin et al. [11]. Nevertheless there are some more refined approaches to get the correct coupling conditions, see Golse [5], Illner and Neunzert [7], Klar [8]. 


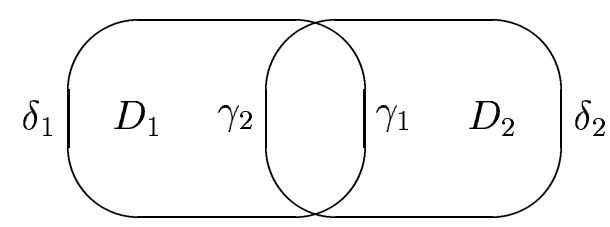

Partial overlapping is allowed and sometimes even necessary. At the inner boundaries $\gamma_{1}, \gamma_{2}$ coupling conditions are defined. At the outer boundaries $\delta_{1}, \delta_{2}$ the usual boundary conditions for the respective equations are used.

We start with a solution of the time dependent aerodynamic equation on $D_{2}$. The boundary condition for the time dependent kinetic equation is then obtained by using a Maxwellian distribution with the macroscopic parameters obtained in $D_{2}$ as ingoing boundary distribution for the domain $D_{1}$ at the boundary $\gamma_{1}$. In turn by the solution on $D_{1}$ we get the macroscopic boundary conditions at $\gamma_{2}$ by simply taking the moments of the Boltzmann distribution as boundary conditions for the macroscopic equation. In analogy to the Marshak method used in transport theory one can also use equality of the fluxes to determine the macroscopic quantities at the boundary $\gamma_{2}$. This procedure has to be repeated until we get finally a coupled solution.

Here the linearized versions of the above equations are considered and the convergence of the iterative scheme is proven:

In section 2 we describe the equations and the domain decomposition scheme.

In section 3 we prove convergence of the above scheme for a linearized Boltzmann equation in $D_{1}$ and a linearized aerodynamic equation in $D_{2}$.

In section 4 we will investigate how far the coupled solution obtained in 3 can be considered as physically correct. It will turn out that in certain situations the overlapping of the domains is crucial.

In the last section we present a numerical simulation coupling two simple kinetic and aerodynamic equations for different mean free paths.

\section{The Domain Decomposition Scheme}

In this section we describe the alternating scheme. To simplify, a 1-dimensional geometry, $x \in[-L, L]$, is considered. In the domain $D_{1}$, i.e. $\left[-L, x_{0}\right]$ the following linearized kinetic equation is solved:

$$
\partial_{t} \varphi+v_{1} \partial_{x} \varphi+\nu(v) \varphi-K \varphi=0
$$

where $\varphi=\varphi(x, v, t), v=\left(v_{1}, \ldots, v_{N}\right) \in S \subseteq \mathbb{R}^{N}, N=1,2,3, t \in[0, \infty)$. We impose the following conditions on $\nu$ and $K$ : 
$C_{2}: \quad K$ is an integraloperator on $\mathcal{L}^{\infty}(S)$ with $K \varphi(v)=\int_{S} k\left(v, v^{\prime}\right) \varphi\left(v^{\prime}\right) d v^{\prime}$, where

$\bar{k}\left(v, v^{\prime}\right) \geq 0 \quad \forall v, v^{\prime} \in S$ and $\int_{S} \bar{k}\left(v, v^{\prime}\right) d v^{\prime} \leq k \forall v \in S, k$ some constant.

For all $\varphi \in \mathcal{L}^{\infty}(S)$ we have $\|K \varphi\|_{\infty} \leq k\|\varphi\|_{\infty}$.

Let now $M$ be the number of linear independent collision invariant, i.e. the number of functions $\psi_{1}, \ldots, \psi_{M}$ with

$$
L\left(\psi_{i}\right)=0, i=1, \ldots, M, \psi_{i} \in \mathcal{L}^{\infty}(S)
$$

where $L$ is defined as

$$
L:=\nu-K
$$

The macroscopic moments are given by

$$
\Theta_{i}(x, t):=\int_{S} \bar{\chi}_{i}(v) \varphi(x, v, t) d v, i=1, \ldots, M
$$

where the $\bar{\chi}_{i}$ are linear independent linear combinations of the $\psi_{i}$ and $\bar{\chi}_{i} \in \mathcal{L}^{\infty}(S)$.

In the domain $D_{2}$, i.e. $\left[-x_{0}, L\right]$ we solve the macroscopic equations for $\Theta=\left(\Theta_{1}, \ldots, \Theta_{M}\right)$ :

$$
\partial_{t} \Theta=A \partial_{x}^{2} \Theta+B \partial_{x} \Theta
$$

with $A, B \in \mathbb{R}^{M \times M}$. We suppose the system to be linear mixed hyperbolic-parabolic with

$$
\begin{aligned}
& A=\left(\begin{array}{cc}
0 & 0 \\
0 & A^{\prime}
\end{array}\right), B=\left(\begin{array}{cc}
B_{11} & B_{12} \\
B_{21} & B_{22}
\end{array}\right), \\
& A^{\prime} \in \mathbb{R}^{(M-L) \times(M-L)} \quad, \quad B_{11} \in \mathbb{R}^{L \times L}, L \leq M .
\end{aligned}
$$

$A^{\prime}$ as well as $B_{11}$ are diagonalizable with Eigenvalues $\lambda_{1}, \ldots \lambda_{L} \in \mathbb{R}$ of $B_{11}$ and Eigenvalues $\lambda_{L+1}, \ldots, \lambda_{M} \in \mathbb{R}, \lambda_{i} \geq \delta>0, i=L+1, \ldots, M$ of $A^{\prime}$.

Moreover we suppose for simplicity an ordering of the $B_{11}$-Eigenvalues:

$$
\lambda_{1}, \ldots, \lambda_{J}=0, \lambda_{J+1}, \ldots, \lambda_{K}>0, \lambda_{K+1}, \ldots, \lambda_{L}<0,0 \leq J \leq K \leq L \leq M
$$

Because $A$ und $B$ are constant we can always change variables in such a way that $A^{\prime}$ and $B_{11}$ are in diagonal form and the general form of the equations remains unchanged. This will be assumed from now on.

Moreover for simplicity we consider only either hyperbolic or parabolic systems. More exactly we impose one of the following conditions on the coefficients $A$ and $B$ :

$C_{3}: \quad L=M$, i.e. $A=0$ and $B=B_{11}$.

$C_{4}: \quad J=K=L=0$, i.e. $A=A^{\prime}$ and $B=B_{22}$. Additionally we assume $B=0$ or alternatively we require that all eigenvalues of $A$ are distinct. 


$$
\varphi(-L, v, t)=f_{+}(v, t), \quad \forall v_{1}>0
$$

and

$$
\Theta_{(J+1 \ldots, K, L+1 \ldots M)}(L, t)=f_{-}(t)
$$

where $f_{+} \in \mathcal{L}^{\infty}(S \times[0, \infty))$ and $f_{-} \in \mathcal{L}^{\infty}\left([0, \infty), \mathbb{R}^{K-J+M-L}\right)$. Here $K-J$ is the number of ingoing characteristic at $x=L$ for the hyperbolic part, $M-L$ is the number of parabolic boundary values and

$$
\Theta_{(J+1 \ldots K, L+1 \ldots M)}(L, t):=\left(\Theta_{J+1}(L, t), \ldots \Theta_{K}(L, t), \Theta_{L+1}(L, t), \ldots, \Theta_{M}(L, t)\right)
$$

The coupling conditions are, according to the discussion in the introduction for our linearized case given by a linearized Maxwellian as the 'distribution function in the macroscopic region'. We generalize to

$$
\varphi_{\text {macro }}(x, v, t):=\sum_{i=1}^{M} \Theta_{i}(x, t) \chi_{i}(v) .
$$

$\chi_{i}, i=1, \ldots, M$ are linear independent linear combinations of the $\psi_{i}, \chi_{i} \in \mathcal{L}^{\infty}(S)$.

This leads to the following coupling conditions at $x_{0}$ and $-x_{0}$ : At the point $x_{0}$ the distribution function going into the kinetic region is given by $\varphi_{\text {macro }}\left(x_{0}, v, t\right)$. The condition is

$$
\varphi\left(x_{0}, v, t\right)=\varphi_{\text {macro }}\left(x_{0}, v, t\right), \quad \forall v_{1}<0 .
$$

On the other side at $-x_{0}$ the boundary values necessary to solve equation $(2.2)$ in $D_{2}$ namely $\Theta_{K+1}, \ldots, \Theta_{M}$ are determined by equalizing the macroscopic fluxes. The conditions are

$$
\begin{aligned}
\int_{v_{1}>0} v_{1} \psi_{j}(v) \varphi\left(-x_{0}, v, t\right) d v & =\int_{v_{1}>0} v_{1} \psi_{j}(v) \sum_{i=1}^{M} \Theta_{i}\left(-x_{0}, t\right) \chi_{i}(v) d v \\
& =\int_{v_{1}>0} v_{1} \psi_{j}(v) \varphi_{\text {macro }}\left(-x_{0}, v, t\right) d v
\end{aligned}
$$

for $j=K+1, \ldots M$. At $-x_{0}$ one therefore has to solve the $(M-K) \times(M-K)$ system of algebraic equations

$$
U\left(\begin{array}{c}
\Theta_{K+1}\left(-x_{0}, t\right) \\
\vdots \\
\Theta_{M}\left(-x_{0}, t\right)
\end{array}\right)=\left(\begin{array}{c}
b_{K+1}(t) \\
\vdots \\
b_{M}(t)
\end{array}\right)
$$

with 


$$
\begin{aligned}
& U_{i j}:=\int_{v_{1}>0} v_{1} \chi_{i}(v) \psi_{j}(v) d v, i, j=K+1, \ldots, M \\
& b_{j}(t):= \int_{v_{1}>0} v_{1} \psi_{j}(v)\left[\varphi\left(-x_{0}, v, t\right)-\sum_{i=1}^{K} \Theta_{i}\left(-x_{0}, t\right) \chi_{i}(v)\right] d v \\
& j=K+1 \ldots M .
\end{aligned}
$$

All integrals are assumed to exist and $U$ must be invertible.

One also has to prescribe initial conditions

$$
\varphi(x, v, 0)=h(x, v) \quad, x \in D_{1}, h \in \mathcal{L}^{\infty}\left(D_{1} \times S\right)
$$

and

$$
\Theta(x, 0)=g(x) \quad, x \in D_{2}, g \in \mathcal{L}^{\infty}\left(D_{2}\right) .
$$

Of course $h$ and $g$ must be compatible with the coupling conditions at the interface as well as with the boundary conditions. For the following again all data are assumed to be as smooth as required and appropriate compatibility conditions at the interface and the boundaries are assumed to be satisfied in order to get a smooth solution in $D_{1}$ and $D_{2}$.

The solutions $\varphi$ and $\Theta$ of equations (2.1) in $D_{1}$ resp. (2.2) in $D_{2}$ with the above boundary and coupling conditions will be called the solution of the coupling problem. It can be found by the following alternating scheme

\section{Initialization:}

Define

$$
\Theta_{(K+1 \ldots M)}^{(0)}\left(-x_{0}, t\right)=\left(\Theta_{K+1}^{(0)}\left(-x_{0}, t\right), \ldots, \Theta_{M}^{(0)}\left(-x_{0}, t\right)\right):=\chi_{+}(t),
$$

where $\chi_{+} \in \mathcal{L}^{\infty}\left([0, \infty), \mathbb{R}^{M-K}\right)$ must be compatible with the initial values.

\section{Iterationstep:}

Let $\Theta_{(K+1 \ldots M)}^{(n)}\left(-x_{0}, t\right)$ be known.

1. Solve equation (2.2) in $D_{2}$ with the initial and boundary values to get $\Theta^{(n)}(x, t) \forall x \in D_{2}$.

2. Determine according to the coupling condition at $x_{0}$

$$
\varphi^{(n+1)}\left(x_{0}, v, t\right):=\sum_{i=1}^{n} \Theta_{i}^{(n)}\left(x_{0}, t\right) \chi_{i}(v), \quad \forall v_{1}<0
$$

3. Solve equation (2.1) in $D_{1}$ with the given inital and boundary conditions to obtain $\varphi^{(n+1)}(x, v, t), \quad \forall x \in D_{1}$. 


$$
\begin{aligned}
& \int_{v_{1}>0} v_{1} \psi_{j}(v) \varphi^{(n+1)}\left(-x_{0}, v, t\right) d v \\
& =\int_{v_{1}>0} v_{1} \psi_{j}(v)\left[\sum_{i=1}^{K} \Theta_{i}^{(n)}\left(-x_{0}, t\right) \chi_{i}(v)+\sum_{i=K+1}^{M} \Theta_{i}^{(n+1)}\left(-x_{0}, t\right) \chi_{i}(v)\right] d v, j=K+1 \ldots M .
\end{aligned}
$$

Example: (The linearized Boltzmann and Euler equations)

In the formulation of Grad the nonlinear Boltzmann equation with a collision operator $Q$ is linearized around a constant Maxwellian state

$$
\bar{M}(v):=\frac{\bar{\rho}}{(2 \pi \bar{T})^{3 / 2}} \exp \left(-\frac{|v-\bar{u}|^{2}}{2 \bar{T}}\right),
$$

where $v \in \mathbb{R}^{3}$ and $\bar{\rho}, \bar{u}=\left(\bar{u}_{1}, \bar{u}_{2}, \bar{u}_{3}\right), \bar{T}$ are constants.

The distribution function $f$ is given as $f=\bar{M}+\bar{M}^{\frac{1}{2}} \varphi \cdot \varphi$ solves the linear problem

$$
\partial_{t} \varphi+v_{1} \partial_{x} \varphi+L \varphi=0
$$

where $L$ is defined as

$$
L \varphi=2 \bar{M}^{-\frac{1}{2}} Q\left(\bar{M}, \bar{M}^{\frac{1}{2}} \varphi\right) .
$$

$L$ can be split into $L=\nu(v)-K$. As proven by Grad [6], $\nu(v) \geq \nu_{0}$ for collision kernels of hard potential intermolecular forces with cut off. For general cut off potentials one can show that

$$
K \varphi=\int_{\mathbb{R}^{3}} \bar{k}\left(v, v^{\prime}\right) \varphi\left(v^{\prime}\right) d v^{\prime}
$$

with

$$
\int_{\mathbb{R}^{3}} \bar{k}\left(v, v^{\prime}\right) d v^{\prime} \leq k \quad \forall v \in \mathbb{R}^{3},
$$

where $v_{0}, k$ are positive constants.

For the linearized Boltzmann collision operator the collision invariants $\psi_{i}, i=0 \ldots 4$ are

$$
\bar{M}^{1 / 2}, \bar{M}^{1 / 2} v_{i}, \bar{M}^{1 / 2}|v|^{2}, \quad i=1,2,3
$$

To first order the asymptotic macroscopic equations associated to the linearized Boltzmann equation for small mean free path are the compressible Euler equations for $\rho, u_{1}, u_{2}$, $u_{3}, T$, linearized around the constant state $\left(\bar{\rho}, \bar{u}_{1}, \bar{u}_{2}, \bar{u}_{3}, \bar{T}\right)$. Eulers equations are hyperbolic. Therefore they meet the above requirements. Concerning the coupling conditions we choose a linearized Maxwellian as the 'macroscopic density function':

$$
\begin{aligned}
\varphi_{\text {macro }}(x, v, t) & =\bar{M}^{1 / 2}(v)\left(\frac{\rho(x, t)}{\bar{\rho}}+\sum_{i=1}^{3} u_{i}(x, t) \frac{\left(v_{i}-\bar{u}_{i}\right)}{\bar{T}}\right. \\
& \left.+T(x, t) \frac{|v-\bar{u}|^{2}-3 \bar{T}}{2 \bar{T}^{2}}\right)
\end{aligned}
$$


Before starting the convergence proof we look at the coupling problem described in section 2 and at the kinetic and aerodynamic equations with fixed boundary values, i.e. equation (2.1) in $D_{1}$ with

$$
\begin{aligned}
\varphi(-L, v, t) & =f_{+}(v, t), v_{1}>0 \\
\varphi\left(x_{0}, v, t\right) & =\chi_{-}(v, t), v_{1}<0 \\
\varphi(x, v, 0) & =h(x, v)
\end{aligned}
$$

where $f_{+}, \chi_{-} \in \mathcal{L}^{\infty}(S \times[0, \infty))$ and equation $(2.2)$ in $D_{2}$ with

$$
\begin{aligned}
\Theta_{(K+1 \ldots M)}\left(-x_{0}, t\right) & =\chi_{+}(t) \\
\Theta_{(J+1 \ldots K, L+1 \ldots M)}(L, t) & =f_{-}(t) \\
\Theta(x, 0) & =g(x),
\end{aligned}
$$

where $f_{-} \in \mathcal{L}^{\infty}\left([0, \infty), \mathbb{R}^{M-L+K-J}\right)$ and $\chi_{+} \in \mathcal{L}^{\infty}\left([0, \infty), \mathbb{R}^{M-K}\right)$.

Using standard arguments as e.g. in Bensoussan et al. [2], p.60, Grad [6], p.53. and Kreiss/Lorenz [9] the following can be shown

Lemma 3.1 Equation (2.1) and (2.2) with the above fixed initial- and boundary conditions have unique solutions $\varphi$ in $\mathcal{L}^{\infty}\left(D_{1} \times S \times[0, T]\right)$ and $\Theta$ in $\mathcal{L}^{\infty}\left(D_{2} \times[0, T], \mathbb{R}^{M}\right), T$ fixed but arbitrary. These solutions are exponentially bounded in $t$, uniformly in $x$ and $v$. They are as smooth as required if the appropriate conditions on the data are imposed.

Concerning the coupling problem there exist unique solutions $\varphi$ in $\mathcal{L}^{\infty}\left(D_{1} \times S \times[0, T]\right)$ and $\Theta$ in $\mathcal{L}^{\infty}\left(D_{2} \times[0, T], \mathbb{R}^{M}\right)$ of the kinetic and aerodynamic equations fulfilling the coupling and boundary conditions.

The convergence proof for the scheme in section 2 will be splitted in several parts:

In the first two steps the above problems with fixed boundary values are more closely investigated. Laplace transformation of the time dependent problem w.r.t. $t$ with a parameter $s+i \omega, s>0, \omega \in \mathbb{R}$ gives in the first step in the kinetic domain, $x \in\left[-L, x_{0}\right]$ the following stationary equation:

$$
(s+i \omega) \hat{\varphi}(x, v, s, w)+v_{1} \partial_{x} \hat{\varphi}+(\nu(v)-K) \hat{\varphi}=h(x, v)
$$

with

$$
\begin{array}{rll}
\hat{\varphi}(-L, v, s, w) & =\hat{f}_{+}(v, s+i \omega) & \forall v_{1}>0 \\
\hat{\varphi}\left(x_{0}, v, s, w\right) & =\hat{\chi}_{-}(v, s+i \omega) & \forall v_{1}<0 .
\end{array}
$$


Rewriting the above equation in integral form one obtains

$$
\hat{\varphi}(x, v, s, \omega)=\varphi_{0}(x, v, s, \omega)+T \hat{\varphi}(x, v, s, \omega)
$$

where

$$
\begin{aligned}
\varphi_{0}(x, v, s, \omega) & :=\left.\hat{f}_{+}(v, s+i \omega) e^{-\frac{x+L}{v_{1}}(\nu+s+i \omega)}\right|_{v_{1}>0} \\
& +\left.\hat{\chi}_{-}(v, s+i \omega) e^{\frac{x_{0}-x}{v_{1}}(\nu+s+i \omega)}\right|_{v_{1}<0} \\
& +\int_{0}^{\tau\left(x, v ;-L, x_{0}\right)} \frac{1}{\nu+s} h\left(x-\frac{\tau^{\prime} v_{1}}{\nu+s}, v\right) e^{-\tau^{\prime}\left(1+\frac{i \omega}{\nu+s}\right)} d \tau^{\prime}
\end{aligned}
$$

and

$$
T \hat{\varphi}(x, v, s, \omega):=\int_{0}^{\tau\left(x, v ;-L, x_{0}\right)} \frac{1}{\nu+s}(K \hat{\varphi})\left(x-\frac{\tau^{\prime} v_{1}}{\nu+s}, v, \omega\right) e^{-\tau^{\prime}\left(1+\frac{i \omega}{\nu+s}\right)} d \tau^{\prime}
$$

with

$$
\tau(x, v ; a, b):= \begin{cases}\frac{x-a}{v_{1}}(\nu+s), & \text { if } v_{1}>0 \\ \frac{x-b}{v_{1}}(\nu+s), & \text { if } v_{1}<0 \\ \infty, & \text { if } v_{1}=0\end{cases}
$$

Lemma 3.2 Let $s$ be fixed and sufficiently large. Equation (3.1) in the integral formulation has for all $\omega$ a unique solution $\hat{\varphi}$ in $\mathcal{L}^{\infty}\left(D_{1} \times S\right) . \hat{\varphi}$ is the Laplace transform of $\varphi$, the solution of (2.1). Moreover we have with some constant $C$, independent of $\omega$

$$
\|\hat{\varphi}\|_{\infty} \leq C\left(\left\|\hat{f}_{+}\right\|_{\infty}+\left\|\hat{\chi}_{-}\right\|_{\infty}+\|h\|_{\infty}\right)
$$

$\|\hat{\varphi}\|_{\infty}$ as a function of $\omega$ is in $\mathcal{L}^{2}(d \omega)$.

Proof: We consider the integral equation. Condition $C_{1}$ gives the boundedness of $\frac{1}{(\nu+s)}$, and hence we obtain $\varphi_{0} \in \mathcal{L}^{\infty}\left(D_{1} \times S\right)$. Using conditions $C_{1}$ and $C_{2}$ we get for all $\omega$

$$
\|T \hat{\varphi}\|_{\infty} \leq\left\|\frac{K}{\nu+s} \hat{\varphi}\right\|_{\infty} \int_{0}^{\infty} e^{-\tau^{\prime}} d \tau^{\prime} \leq \frac{k}{\nu_{0}+s}\|\hat{\varphi}\|_{\infty} \quad \forall \hat{\varphi} \in \mathcal{L}^{\infty}\left(D_{1} \times S\right) .
$$

In particular $T: \mathcal{L}^{\infty}\left(D_{1} \times S\right) \rightarrow \mathcal{L}^{\infty}\left(D_{1} \times S\right)$ is a bounded linear operator and $\hat{\varphi} \rightarrow \varphi_{0}+T \hat{\varphi}$ is a contraction mapping in $\mathcal{L}^{\infty}\left(D_{1} \times S\right)$, if $s>k-\nu_{0}$. By the contraction mapping theorem there is a unique solution $\hat{\varphi}=(I-T)^{-1} \varphi_{0}$ of our integral equation. This proves the first statement and also the estimate since $\left\|(I-T)^{-1}\right\|_{\infty} \leq\left(1-\frac{k}{\nu_{0}+s}\right)^{-1}$ and $\left\|\varphi_{0}\right\|_{\infty} \leq \tilde{C}, \tilde{C}$ some constant. The remaining statement is evident using Lemma 3.1. and the properties of Fourier- and Laplace transforms. 


$$
\begin{array}{rll}
(s+i \omega) \Theta=A \partial_{x}^{2} \Theta+B \partial_{x} \Theta & +g \\
\hat{\Theta}_{(K+1 \ldots M)}\left(-x_{0}\right)=\hat{\chi}_{+}(s+i \omega) & \in \mathbb{R}^{M-K} \\
\hat{\Theta}_{(J+1 \ldots K, L+1 \ldots M)}(L)=\hat{f}_{-}(s+i \omega) & \in \mathbb{R}^{M-L+K-J}
\end{array}
$$

Using conditions $C_{3}$ or $C_{4}$ with $B=0$ and the diagonal form of the coefficient matrices $A^{\prime}$ and $B_{11}$ one can prove the following lemma by solving equation (3.2) explicitely. If $C_{4}$ is valid with $B$ not equal to zero the proof is achieved for large s by a perturbation argument.

Lemma 3.3 Let $s$ be fixed and sufficiently large.

Equation (3.2) has a unique smooth solution $\hat{\Theta}$ in $\mathcal{L}^{\infty}\left(D_{2}, \mathbb{R}^{M}\right)$, the Laplace transform with parameter $s+i \omega$ of $\Theta$ found in Lemma 3.1. Moreover with $C$ a constant

$$
\|\hat{\Theta}\|_{\infty} \leq C\left(\left\|\hat{\chi}_{+}\right\|_{\infty}+\left\|\hat{f}_{-}\right\|_{\infty}+\|g\|_{\infty}\right) .
$$

$\|\hat{\Theta}\|_{\infty}$ as a function of $\omega$ is in $\mathcal{L}^{2}(d \omega)$.

The second step in the convergence proof will be to prove some additional properties of the solution of the above stationary equations on the domains $D_{1}$ and $D_{2}$.

Let $\hat{\varphi}$ be the solution of (3.1) in $D_{1}$ with the above boundary values.

$$
A_{-x_{0}}^{1}: \mathcal{L}^{\infty}\left(S^{-}\right) \rightarrow \mathcal{L}^{\infty}\left(S^{+}\right)
$$

is defined $\forall x_{0} \in[0, L),\left.\hat{\chi}_{-}\right|_{v_{1}<0} \in \mathcal{L}^{\infty}\left(S^{-}\right)$as

$$
A_{-x_{0}}^{1}:\left.\left.\hat{\chi}_{-}(v)\right|_{v_{1}<0} \rightarrow \hat{\varphi}\left(-x_{0}, v\right)\right|_{v_{1}>0}
$$

Then

Lemma 3.4 $A_{-x_{0}}^{1}$ is a contraction mapping, if the parameter $s$ in equation (3.1) is chosen sufficiently large. The Lipschitz constant $\sigma_{1}$ is independent of $\omega \in \mathbb{R}$ and as small as desired, if $s$ is large enough.

Proof: We define $\forall x \in\left[-L, x_{0}\right]$

$$
\hat{\psi}(x, v):=\hat{\varphi}(x, v)-\left.e^{-\frac{\left(x-x_{0}\right)}{v_{1}}(\nu+s+i \omega)} \hat{\chi}_{-}(v, s+i \omega)\right|_{v_{1}<0} .
$$

Then we have

$$
\left.\hat{\psi}(x, v)\right|_{v_{1}>0}=\left.\hat{\varphi}(x, v)\right|_{v_{1}>0}
$$

and especially

$$
A_{-x_{0}}^{1}\left(\left.\hat{\chi}_{-}\right|_{v_{1}<0}\right)=\left.\hat{\psi}\left(-x_{0}, v\right)\right|_{v_{1}>0} .
$$




$$
\begin{aligned}
\hat{\psi}(x, v) & =\left.\hat{f}_{+}(v) e^{\frac{-L-x}{v_{1}}(\nu+s+i \omega)}\right|_{v_{1}>0} \\
& +\int_{\tau\left(x, v ;-L, x_{0}\right)} \frac{1}{\nu+s} h\left(x-\frac{\tau^{\prime} v_{1}}{\nu+s}, v\right) e^{-\tau^{\prime}\left(1+\frac{i \omega}{\nu+s}\right)} d \tau^{\prime} \\
& +T\left(\hat{\psi}+\left.e^{-\frac{\left(x-x_{0}\right)}{v_{1}}(\nu+s+i \omega)} \hat{\chi}_{-}(v)\right|_{v_{1}<0}\right) .
\end{aligned}
$$

If $\hat{\varphi}$ and $\tilde{\varphi}$ are the solutions of (3.1) in $D_{1}$ for 2 different boundary values $\hat{\chi}_{-}$and $\tilde{\chi}_{-}$at $x=x_{0}$ and $\tilde{\psi}$ is defined analogously to $\hat{\psi}$ then we obtain:

$$
\begin{aligned}
\|\hat{\psi}-\tilde{\psi}\|_{\infty} & \leq\|T(\hat{\psi}-\tilde{\psi})\|_{\infty}+\left\|T\left(e^{-\frac{\left(x-x_{0}\right)}{v_{1}}(\nu+s+i \omega)}\left(\left.\hat{\chi}_{-}\right|_{v_{1}<0}-\left.\tilde{\chi}_{-}\right|_{v_{1}<0}\right)\right)\right\|_{\infty} \\
& \leq\|T\|_{\infty}\|\hat{\psi}-\tilde{\psi}\|_{\infty}+\|T\|_{\infty}\left\|\left.\hat{\chi}_{-}\right|_{v_{1}<0}-\left.\tilde{\chi}_{-}\right|_{v_{1}<0}\right\|_{\infty},
\end{aligned}
$$

where $\|\cdot\|_{\infty}$ is the norm in $\mathcal{L}^{\infty}\left(\left[-L, x_{0}\right] \times S\right)$ resp. $\mathcal{L}^{\infty}(S)$. This gives

$$
\begin{aligned}
& \|\hat{\psi}-\tilde{\psi}\|_{\infty} \leq \frac{\|T\|_{\infty}}{1-\|T\|_{\infty}}\left\|\left.\hat{\chi}_{-}\right|_{v_{1}<0}-\left.\tilde{\chi}_{-}\right|_{v_{1}<0}\right\|_{\infty} \\
& \leq \sigma_{1}\left\|\left.\hat{\chi}_{-}\right|_{v_{1}<0}-\left.\tilde{\chi}_{-}\right|_{v_{1}<0}\right\|_{\infty}
\end{aligned}
$$

where $\sigma_{1}:=\frac{\|T\|_{\infty}}{1-\mid T \|_{\infty}}$. With $\|T\|_{\infty} \leq \frac{k}{\nu_{0}+s}$ (compare the proof of Lemma 3.2) we get $\sigma_{1}<\frac{k}{\nu_{0}+s-k}$. Taking at least $s>2 k-\nu_{0}$ we obtain $\sigma_{1}<1$. Then especially

$$
\begin{aligned}
\|\left.\hat{\psi}\left(-x_{0}, v\right)\right|_{v_{1}>0} & -\left.\tilde{\psi}\left(-x_{0}, v\right)\right|_{v_{1}>0} \|_{\infty} \\
& \leq \sigma_{1}\left\|\left.\hat{\chi}_{-}\right|_{v_{1}<0}-\left.\tilde{\chi}_{-}\right|_{v_{1}<0}\right\|_{\infty}
\end{aligned}
$$

and consequently

$$
\begin{aligned}
\| A_{-x_{0}}^{1}\left(\left.\hat{\chi}_{-}\right|_{v_{1}<0}\right) & -A_{-x_{0}}^{1}\left(\left.\tilde{\chi}_{-}\right|_{v_{1}<0}\right) \|_{\infty} \\
& \leq \sigma_{1}\left\|\left.\hat{\chi}_{-}\right|_{v_{1}<0}-\left.\tilde{\chi}_{-}\right|_{v_{1}<0}\right\|_{\infty}, \forall x_{0} \in[0, L)
\end{aligned}
$$

where $\sigma_{1}$ is smaller than 1 if $\mathrm{s}$ is large.

Let $\hat{\Theta}$ be the above solution of (3.2) in $D_{2}$. The Albedo operator $A_{x_{0}}^{2}$ is for fixed $s$ and $\omega$ defined as

$$
\begin{aligned}
A_{x_{0}}^{2}: & \mathbb{R}^{M-K} \rightarrow \mathbb{R}^{M} \\
& \hat{\chi}_{+} \rightarrow \hat{\Theta}\left(x_{0}\right) \quad \forall x_{0} \in[0, L)
\end{aligned}
$$

The main property is 


\section{Proof:}

In the hyperbolic case with $B=B_{11}$, as well as in the parabolic case with $B=B_{22}=0$, the lemma can be proven by using the diagonal form of $B=B_{11}$ respectively $A=A^{\prime}$ and solving the equations explicitely.

In the parabolic case with $B \neq 0$ we can treat the system as a small perturbation of the one with $B=0$ if $s$ is large. Using the fact that all Eigenvalues of $A$ are distinct the lemma can be proven in this case as well.

In the third step of the proof we return to the iteration scheme and consider the Laplace transforms $\hat{\varphi}^{(n)}(x, v), \hat{\varphi}(x, v), x \in D_{1}$ and $\hat{\Theta}^{(n)}(x), \hat{\Theta}(x), x \in D_{2}$ of $\varphi^{(n)}(x, v, t), \varphi(x, v, t)$ and $\Theta^{(n)}(x, t), \Theta(x, t)$ defined in Section 2 .

Lemma 3.6 Let $s$ be fixed and sufficiently large and $\omega \in \mathbb{R}$.

The sequence $\hat{\Theta}^{(n)}$ converges as $n \rightarrow \infty$ to $\hat{\Theta}$ in $\mathcal{L}^{\infty}\left(D_{2}, \mathbb{R}^{M}\right)$. The sequence $\hat{\varphi}^{(n)}$ converges to $\hat{\varphi}$ in $\mathcal{L}^{\infty}\left(D_{1} \times S\right)$. Moreover

$$
\begin{aligned}
\left\|\hat{\varphi}^{(n)}-\hat{\varphi}\right\|_{\infty} & \leq \sigma^{n} H_{1}(\omega) \\
\left\|\hat{\Theta}^{(n)}-\hat{\Theta}\right\|_{\infty} & \leq \sigma^{n} H_{2}(\omega)
\end{aligned}
$$

where $\sigma<1$ is independent of $\omega$ and $H_{i}, i=1,2$ is in $\mathcal{L}^{2}(d \omega)$.

\section{Proof:}

We start the proof by considering the values at the interface: To formulate the following more easily we define $\forall x_{0} \in[0, L)$

$$
\begin{aligned}
A_{-x_{0}}^{2, K}: & \mathbb{R}^{M-K} \rightarrow \mathbb{R}^{K} \\
& \hat{\chi}_{+} \rightarrow \hat{\Theta}_{(1 \ldots K)}\left(-x_{0}\right)
\end{aligned}
$$

where $\hat{\Theta}$ is again the solution of $(3.2)$ in $D_{2}$ with $\hat{\Theta}_{(K+1, \ldots, M)}\left(-x_{0}\right)=\hat{\chi}_{+}$. We get with the simple form of the hyperbolic part of the system $\forall x_{0} \in[0, L)$ :

$$
A_{-x_{0}}^{2, K}\left(\hat{\chi}_{+}\right)-A_{-x_{0}}^{2, K}\left(\tilde{\chi}_{+}\right)=0 .
$$

Remembering the iteration scheme and in particular (2.3) we see that the boundary values $\hat{\Theta}_{(K+1 \ldots M)}^{(n+1)}\left(-x_{0}\right)$ at $-x_{0}$ fulfill 


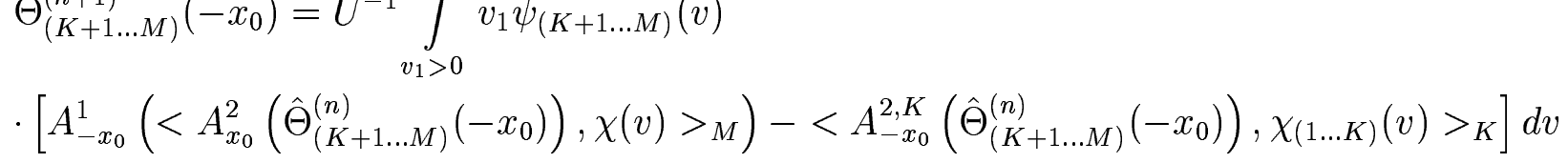

where $<,>_{M},<,>_{K}$ denote the usual scalar products in $\mathbb{R}^{M}, \mathbb{R}^{K}, \chi:=\left(\chi_{1}, \ldots, \chi_{M}\right)$, $\chi_{(1 \ldots K)}:=\left(\chi_{1} \ldots, \chi_{K}\right)$, and $\psi_{(K+1 \ldots M)}:=\left(\psi_{K+1}, \ldots, \psi_{M}\right)$.

With

$$
x^{(n)}:=\hat{\Theta}_{(K+1 \ldots M)}^{(n)}\left(-x_{0}\right)
$$

we get

$$
\begin{aligned}
& x^{(n+1)}=U^{-1} \int_{v_{1}>0} v_{1} \psi_{(K+1 \ldots M)}(v) \\
& \cdot\left[A_{-x_{0}}^{1}\left(<A_{x_{0}}^{2}\left(x^{(n)}\right), \chi(v)>_{M}\right)-<A_{-x_{0}}^{2, K}\left(x^{(n)}\right), \chi_{(1 \ldots K)}(v)>_{K}\right] d v
\end{aligned}
$$

In short:

$$
x^{(n+1)}=T x^{(n)} .
$$

We show that $T$ is a contraction mapping in $\mathbb{R}^{M-K}$ :

Let $x, y \in \mathbb{R}^{M-K}, C$ be a generic constant and $\|\cdot\|_{\infty}$ the norm in $\mathcal{L}^{\infty}(S)$. Let $\sigma_{1}$ denote the Lipschitzconstant of $A_{-x_{0}}^{1}$ according to Lemma $3.4, \sigma_{2}$ the one of $A_{x_{0}}^{2}$ according to Lemma 3.5.Then

$$
\begin{aligned}
& |T x-T y| \leq C \| A_{-x_{0}}^{1}\left(<A_{x_{0}}^{2}(x), \chi(v)>_{M}\right)-<A_{-x_{0}}^{2, K}(x), \chi_{(1 \ldots K)}(v)>_{K} \\
& -A_{-x_{0}}^{1}\left(<A_{x_{0}}^{2}(y), \chi(v)>_{M}\right)+<A_{-x_{0}}^{2, K}(y), \chi_{(1 \ldots K)}(v)>_{K} \|_{\infty} \\
& \leq C \cdot \sigma_{1}\left\|<A_{x_{0}}^{2}(x), \chi(v)>_{M}-<A_{x_{0}}^{2}(y), \chi(v)>_{M}\right\|_{\infty} \\
& +C \cdot\left\|<A_{-x_{0}}^{2, K}(x), \chi_{(1 \ldots K)}(v)>_{K}-<A_{-x_{0}}^{2, K}(y), \chi_{(1 \ldots K)}(v)>_{K}\right\|_{\infty} \\
& \leq C \cdot \sigma_{1}\left|A_{x_{0}}^{2}(x)-A_{x_{0}}^{2}(y)\right| \cdot\|\chi\|_{\infty} .
\end{aligned}
$$

This is smaller than

$$
C \cdot \sigma_{1}|x-y| \quad \text { if } \quad x_{0}=0
$$

and smaller than

$$
C \cdot \sigma_{1} \sigma_{2}|x-y| \text { if } x_{0} \neq 0,
$$

$C$ some constant. Choosing $s$ sufficiently large, we see that $\sigma:=\sigma_{1} C$ or $\sigma:=\sigma_{1} \sigma_{2} C$ is smaller than 1 , which gives the contraction property. We remark that for $x_{0}=0$ the operator $A_{x_{0}}^{2}$ does not give any contribution to the contraction.

By the contraction mapping theorem $\hat{\Theta}_{(K+1 \ldots M)}^{(n)}\left(-x_{0}\right)$ converges to a limit in $\mathbb{R}^{M-K}$. This limit is $\hat{\Theta}_{(K+1 \ldots M)}\left(-x_{0}\right)$. Using the same arguments one can then prove, that 


$$
\begin{aligned}
& \left\|\left.\hat{\varphi}^{(n+1)}\left(x_{0}, v\right)\right|_{v_{1}<0}-\left.\hat{\varphi}^{(n)}\left(x_{0}, v\right)\right|_{v_{1}<0}\right\|_{\infty} \\
& \leq \sigma\left\|\left.\hat{\varphi}^{(n)}\left(x_{0}, v\right)\right|_{v_{1}<0}-\left.\hat{\varphi}^{(n-1)}\left(x_{0}, v\right)\right|_{v_{1}<0}\right\|_{\infty}
\end{aligned}
$$

and therefore

$$
\begin{aligned}
& \left\|\left.\hat{\varphi}^{(n)}\left(x_{0}, v\right)\right|_{v_{1}<0}-\left.\hat{\varphi}\left(x_{0}, v\right)\right|_{v_{1}<0}\right\|_{\infty} \\
& \leq \frac{\sigma^{n}}{1-\sigma}\left\|\left.\hat{\varphi}^{(1)}\left(x_{0}, v\right)\right|_{v_{1}<0}-\left.\hat{\varphi}^{(0)}\left(x_{0}, v\right)\right|_{v_{1}<0}\right\|_{\infty}
\end{aligned}
$$

where $\sigma<1$ is independent of $\omega \in \mathbb{R}$.

Remarking, that $\left(\hat{\varphi}^{(n)}-\hat{\varphi}\right)$ are solutions of $(3.1)$ in $D_{1}$ with initial values equal to 0 , boundary values at $-L$ equal to 0 and boundary values at $x_{0}$ equal to

$$
\left.\hat{\varphi}^{(n)}\left(x_{0}, v\right)\right|_{v_{1}<0}-\left.\hat{\varphi}\left(x_{0}, v\right)\right|_{v_{1}<0}
$$

we have, using Lemma 3.2:

$$
\left\|\hat{\varphi}^{(n)}-\hat{\varphi}\right\|_{\infty} \leq\left. C|| \hat{\varphi}^{(n)}\left(x_{0}, v\right)\right|_{v_{1}<0}-\left.\hat{\varphi}\left(x_{0}, v\right)\right|_{v_{1}<0} \|_{\infty},
$$

$\mathrm{C}$ some constant independent of $\omega$ and $n$. $\|\cdot\|_{\infty}$ denotes here the norm in $\mathcal{L}^{\infty}\left(D_{1} \times S\right)$ respectively $\mathcal{L}^{\infty}(S)$. All together

$$
\begin{aligned}
\left\|\hat{\varphi}^{(n)}-\hat{\varphi}\right\|_{\infty} & \leq C \cdot \sigma^{n}\left\|\left.\hat{\varphi}^{(1)}\left(x_{0}, v\right)\right|_{v_{1}<0}-\left.\hat{\varphi}^{(0)}\left(x_{0}, v\right)\right|_{v_{1}<0}\right\|_{\infty} \\
& \leq C \cdot \sigma^{n}\left\|\hat{\varphi}^{(1)}-\hat{\varphi}^{(0)}\right\|_{\infty} \\
& \leq C \cdot \sigma^{n}\left(\left\|\hat{\varphi}^{(1)}\right\|_{\infty}+\left\|\hat{\varphi}^{(0)}\right\|_{\infty}\right),
\end{aligned}
$$

where $C$ denotes a constant. This gives the convergence of $\hat{\varphi}^{(n)}$ to $\hat{\varphi}$. Moreover by Lemma 3.2 we know that $\left\|\hat{\varphi}^{(0)}\right\|_{\infty}$ as well as $\left\|\hat{\varphi}^{(1)}\right\|_{\infty}$ are in $\mathcal{L}^{2}(d \omega)$, which finishes the proof for the domain $D_{1}$. We use the same arguments for the domain $D_{2}$.

Finally in the last step the following can be shown

Theorem 3.7 For the sequences $\varphi^{(n)}(x, v, t)$ and $\Theta^{(n)}(x, t)$ of iterative solutions of (2.1) and (2.2) the following is true:

$\varphi^{(n)}$ converges in $\mathcal{L}^{\infty}\left(D_{1} \times S, \mathcal{L}^{2}[0, T]\right)$ to $\varphi$.

$\Theta^{(n)}$ converges in $\mathcal{L}^{\infty}\left(D_{2}, \mathcal{L}^{2}\left([0, T], \mathbb{R}^{M}\right)\right)$ to $\Theta$.

Here $T$ is finite but arbitrary. The convergence is geometric. $\varphi, \Theta$ is the unique solution of the coupling problem. 


$$
\begin{aligned}
& \left\|\int_{-\infty}^{\infty}\left|\hat{\varphi}^{(n)}(x, v, s, \omega)-\hat{\varphi}(x, v, s, \omega)\right|^{2} d \omega\right\|_{\infty} \\
& \leq \int_{-\infty}^{\infty}\left\|\hat{\varphi}^{(n)}(x, v, s, \omega)-\hat{\varphi}(x, v, s, \omega)\right\|_{\infty}^{2} d \omega \leq \int_{-\infty}^{\infty}\left(\sigma^{2}\right)^{n}\left(H_{1}(\omega)\right)^{2} d \omega \\
& \leq C \sigma^{n}
\end{aligned}
$$

$\sigma<1, C$ some constant and $\|\cdot\|_{\infty}$ the norm in $\mathcal{L}^{\infty}\left(D_{1} \times S\right)$. Using Parseval's relation for a function and its Fourier transform on $\mathcal{L}^{2}(\mathbb{R})$ and rewriting it in terms of the Laplace transform gives in this case for arbitrary $s>0$

$$
\begin{aligned}
& \int_{0}^{T}\left|\varphi^{(n)}(x, v, t)-\varphi(x, v, t)\right|^{2} d t \\
& \leq \frac{e^{2 s T}}{2 \pi} \int_{-\infty}^{\infty}\left|\hat{\varphi}^{(n)}(x, v, s, \omega)-\hat{\varphi}(x, v, s, \omega)\right|^{2} d \omega \quad \forall x \in D_{1}, v \in S
\end{aligned}
$$

Therefore choosing $s$ fixed but large enough, one obtains

$$
\begin{aligned}
& \left\|\int_{0}^{T}\left|\varphi^{(n)}(x, v, t)-\varphi(x, v, t)\right|^{2} d t\right\|_{\infty} \leq e^{2 s T} \cdot C\left\|\int_{-\infty}^{\infty}\left|\hat{\varphi}^{(n)}(x, v, s, \omega)-\hat{\varphi}(x, v, s, \omega)\right|^{2} d \omega\right\|_{\infty} \\
& \leq C \cdot e^{2 s T} \sigma^{n} \\
& \leq C \sigma^{n} \quad \text { with } \quad \sigma<1, C \text { a generic constant. }
\end{aligned}
$$

This means $\varphi^{(n)}$ converges in $\mathcal{L}^{\infty}\left(D_{1} \times S, \mathcal{L}^{2}[0, T]\right)$ geometrically to $\varphi$.

The same arguments are used to show that $\Theta^{(n)}$ converges to $\Theta$.

Remark : It is not clear whether the obtained coupled solution is physically meaningful. We investigate this point further in the next section considering a special situation.

\section{The Physical Correctness of the Coupled Solution}

We restrict ourselves in this section to linearized kinetic equations with only one collision invariant, e.g. neutron transport equations. The aerodynamic equation will be a diffusion equation. The following situation may occur however as well for the coupling of the full Boltzmann equation with the Navier-Stokes equations. 


$$
K \varphi(x, v, t)=\int_{S} \bar{k}\left(v, v^{\prime}\right) \varphi\left(x, v^{\prime}, t\right) d v
$$

with $\bar{k}$ rotationally invariant, symmetric in $v$ and $v^{\prime}, k_{2}>\bar{k}\left(v, v^{\prime}\right)>k_{1}>0, k_{1}, k_{2}$ constants and $\int_{S} \bar{k}\left(v, v^{\prime}\right) d v^{\prime}=1$. With these assumptions one can show that the constants are the only collision invariants of $L=I-K$. In particular all conditions imposed on $\nu$ and $K$ in Section 2 are fulfilled. We consider the kinetic equation

$$
\partial_{t} \varphi_{\epsilon}+v_{1} \partial_{x} \varphi_{\epsilon}+\frac{1}{\epsilon}\left(\varphi_{\epsilon}-K \varphi_{\epsilon}\right)=0
$$

where $\epsilon>0$ is the mean free path and rescale the time variable $t^{\prime}=t \epsilon$. Writing again $t$ instead of $t^{\prime}$ this results in

$$
\partial_{t} \varphi_{\epsilon}+\frac{1}{\epsilon} v_{1} \partial_{x} \varphi_{\epsilon}+\frac{1}{\epsilon^{2}}\left(\varphi_{\epsilon}-K \varphi_{\epsilon}\right)=0
$$

There is only one macroscopic quantity $\Theta=\int_{S} \varphi_{\epsilon}(x, v, t) d v$, which fulfills for small $\epsilon$ approximately the macroscopic equation associated to (4.1), the diffusion equation

$$
\partial_{t} \Theta=D \cdot \partial_{x}^{2} \Theta
$$

$D>0$ a constant related to $K$. According to the well known 'Diffusion Approximation Theorem', see e.g. Bardos et al [1] or Bensoussan et al [2], the solution of the kinetic equation (4.1) will tend for $\epsilon \rightarrow 0$ to the solution of (4.2).

We assume the physical situation to be described by the kinetic equation (4.1) with different mean free paths $\epsilon$ in $[-L, 0]$ and $[0, L]$. In $[0, L]$ we suppose $\epsilon$ to be small such that the diffusion approximation, i.e. equation (4.2), is valid. Decomposing the domain $[-L, L]$ into two overlapping subdomains $D_{1}=\left[-L, x_{0}\right]$ and $D_{2}=\left[-x_{0}, L\right]$ and considering the coupling of (4.1) in $D_{1}$ and (4.2) in $D_{2}$ with coupling conditions given in Section 2, we want to obtain a coupled solution that approximates the true kinetic solution. I.e. the reference solution to which this coupled solution must be compared is the solution of the kinetic equation (4.1) in the whole domain with different parameters $\epsilon$ in $D_{1}$ and $D_{2}$, where $\epsilon$ is small in $D_{2}$. A numerical example with different parameters $\epsilon$ in $D_{1}$ is shown in Section 5.

In this section we test the validity of the coupled solution by considering a situation with $\epsilon$ tending to 0 also in $D_{1}$. In this case the kinetic reference solution in the whole domain will tend to the solution of the aerodynamic equation on $[-L, L]$. The same is expected for the coupled solution. We say the coupling is physically correct or better physically reasonable if in this case the coupled solution is approximated by the solution of the macroscopic equation (4.2) in the whole domain $[-L, L]$. 


$$
\varphi_{\epsilon}(-L, v, t)=f_{+}(t) \quad, v_{1}>0
$$

where $f_{+}$is independent of $v$

$$
\Theta(L, t)=f_{-}(t)
$$

$f_{+}, f_{-} \in \mathcal{L}^{\infty}([0, \infty))$. The initial conditions are

$$
\begin{aligned}
\varphi_{\epsilon}(x, v, 0) & =g(x) \quad \forall x \in D_{1}, \\
\Theta(x, 0) & =g(x) \quad \forall x \in D_{2},
\end{aligned}
$$

$g \in \mathcal{L}^{\infty}([-L, L])$ where the initial condition for $\varphi_{\epsilon}$ is independent of $v$ as indicated. The coupling conditions are according to Section 2 simply

$$
\varphi_{\epsilon}\left(x_{0}, v, t\right)=\Theta\left(x_{0}, t\right) \quad v_{1}<0
$$

and

$$
\int_{v_{1}>0} v_{1} \varphi_{\epsilon}\left(-x_{0}, v, t\right) d v=\int_{v_{1}>0} v_{1} \Theta\left(-x_{0}, t\right) d v
$$

As before all data are assumed to be smooth. As usual the boundary-, coupling- and initial conditions must be compatible with each other in order to get smooth solutions in the two domains $D_{1}, D_{2}$. Realizing that $\Theta$ depends on $\epsilon$ in $D_{1}$ by the coupling conditions we write $\Theta_{\epsilon}$.

Existence and uniqueness of the solution of the coupling problem follow from Lemma 3.1.

The iteration scheme to find this solution is:

\section{Initialization:}

Define $\Theta_{\epsilon}^{(0)}\left(-x_{0}, t\right)=\chi_{+}(t)$ independent of $\epsilon$, compatible with the initial conditions.

\section{Iteration step:}

Let $\Theta_{\epsilon}^{(n)}\left(-x_{0}, t\right)$ be known

1. Solve (4.2) in $D_{2}$ to get $\Theta_{\epsilon}^{(n)}(x, t), x \in D_{2}$

2. Determine $\left.\varphi_{\epsilon}^{(n+1)}\left(x_{0}, v, t\right)\right|_{v_{1}<0}=\Theta_{\epsilon}^{(n)}\left(x_{0}, t\right)$

3. Solve (4.1) in $D_{1}$ to obtain $\varphi_{\epsilon}^{(n+1)}(x, v, t), x \in D_{1}$

4. Determine $\Theta_{\epsilon}^{(n+1)}\left(-x_{0}, t\right)=\frac{\int_{v_{1}>0} v_{1} \varphi_{\epsilon}^{(n+1)}\left(-x_{0}, v, t\right) d v}{\int_{v_{1}>0} v_{1} d v}$ 
solution, that is the global solution $\Theta_{G}(x, t)$ of $(4.2)$ in the whole domain $[-L, L]$ with

$$
\begin{aligned}
\Theta_{G}(-L, t) & =f_{+}(t) \\
\Theta_{G}(L, t) & =f_{-}(t) \\
\Theta_{G}(x, 0) & =g(x) .
\end{aligned}
$$

First of all we consider an example with nonoverlapping domains i.e. $x_{0}=0$,

$$
D_{1}=[-L, 0], D_{2}=[0, L] .
$$

We take the boundary conditions

$$
\begin{aligned}
\varphi_{\epsilon}^{(n)}(-L, v, t) & =f_{+} \\
\Theta_{\epsilon}^{(n)}(L, t) & =f_{-}
\end{aligned}
$$

to be time independent and the initial conditions

$$
\begin{aligned}
\varphi_{\epsilon}^{(n)}(x, v, 0) & =f_{+}, \\
\Theta_{\epsilon}^{(n)}(x, 0) & =\frac{f_{-}-f_{+}}{L} x+f_{+} .
\end{aligned}
$$

The initialization is $\chi_{+}(t):=f_{+}$. For all $\epsilon$ the limiting value of the iteration can be given explicitely:

$$
\begin{aligned}
\varphi_{\epsilon}(x, v, t) & =f_{+}, \forall x \in D_{1} \\
\Theta_{\epsilon}(x, t) & =\frac{f_{-}-f_{+}}{L} x+f_{+} \quad \forall x \in D_{2} .
\end{aligned}
$$

Considering in contrast the solution of $(4.2)$ in $[-L, L]$ with these boundary- and initial values we can easily get the following result:

$\Theta_{G}(x, t)$ is not constant w.r.t. $t$. It tends for large $t$ to $\Theta_{G}(x, t)=\frac{f_{-}-f_{+}}{2 L}(x+L)+f_{+}$for all $x \in[-L, L]$.

This means that when $\epsilon$ is small the coupled solution is not close to the solution of the diffusion equation in the whole domain, the coupling does not yield a physically reasonable solution. In the following we show that such a situation cannot occur for overlapping domains, i.e. $x_{0} \neq 0, x_{0} \in(0, L)$.

Remark: The coupling of two diffusion equations requires the overlapping of domains or differentiability conditions at the interface to ensure uniqueness of the coupled solution. In the present case however we get a unique coupled solution for either overlapping 
Let $\varphi_{\epsilon}^{(n)}$ be the smooth iterative solutions of (4.1) in $D_{1}$. We have

$$
\begin{aligned}
\varphi_{\epsilon}^{(n)}(-L, v, t) & =f_{+}(t), v_{1}>0 \\
\varphi_{\epsilon}^{(n)}(x, v, 0) & =g(x)
\end{aligned}
$$

and according to the coupling conditions

$$
\varphi_{\epsilon}^{(n)}\left(x_{0}, v, t\right)=\Theta_{\epsilon}^{(n-1)}\left(x_{0}, t\right), v_{1}<0 .
$$

We remark that $f_{+}, g$ and $\Theta_{\epsilon}^{(n-1)}\left(x_{0}, t\right)$ are independent of $v$. Let $\Theta_{\epsilon, A}^{(n)}$ be the solution of (4.2) in $D_{1}$ with the same boundary and initial conditions. Then:

Lemma 4.1 (The Diffusion Approximation)

$$
\left|\varphi_{\epsilon}^{(n)}(x, v, t)-\Theta_{\epsilon, A}^{(n)}(x, t)\right| \leq \epsilon C \quad \forall t \in[0, T], x \in D_{1}, v \in S,
$$

where the constant $C$ is independent of $n, \epsilon, x, v$ and $t$.

Proof: For boundary conditions at $x_{0}$ independent of $n$ and $\epsilon$ the proof is classical, see e.g. Bardos et al [1] or Bensoussan et al [2]. To get the bound $C$ independent of $n$ and $\epsilon$ we must have a closer look at the expansion procedure leading to the diffusion approximation. By following the expansion one can see, that the constant $C$ is determined by bounds on the values of the derivatives of $\Theta_{\epsilon, A}^{(n)}$ up to second order. The uniform boundedness in $n, \epsilon, x \in D_{1}$ and $t \in[0, T]$ of these derivatives is shown by considering the iteration scheme and using the maximum principles for parabolic and transport equations in the respective domains in every iteration step. This will be sufficient to prove the lemma.

Using the overlapping of the regions it is now possible to prove

Theorem 4.2 Let $x_{0} \neq 0, x_{0} \in(0, L)$. Let $\varphi_{\epsilon}, \Theta_{\epsilon}$ be the solution of the coupling problem obtained by the iteration scheme.

As $\epsilon$ tends to $0 \varphi_{\epsilon}, \Theta_{\epsilon}$ will tend to the solutions of the diffusion equation $\Theta_{G}$ in the whole domain $[-L, L]$ :

$$
\begin{aligned}
& \left\|\varphi_{\epsilon}-\Theta_{G}\right\| \leq C \epsilon \\
& \left\|\Theta_{\epsilon}-\Theta_{G}\right\| \leq C \epsilon
\end{aligned}
$$

where $\|\cdot\|$ is the norm in $\mathcal{L}^{\infty}\left(D_{1} \times S, \mathcal{L}^{2}[0, T]\right)$ respectively $\mathcal{L}^{\infty}\left(D_{2}, \mathcal{L}^{2}[0, T]\right)$ and $C$ is a constant. 
differences of boundary values

$$
\sup _{t \in[0, T]}\left|\Theta_{\epsilon}^{(n+1)}\left(-x_{0}, t\right)-\Theta_{G}\left(-x_{0}, t\right)\right|
$$

where $\Theta_{\epsilon}^{(n)}$ are the solutions of the diffusion equation in $D_{2}$ of the alternating scheme and $\Theta_{G}$ is the global solution of $(4.2)$ in $[-L, L]$. Let $\Theta_{\epsilon, A}^{(n)}$ be the diffusion approximation for $\varphi_{\epsilon}^{(n)}$ in $D_{1}$ according to Lemma 4.1:

$$
\begin{aligned}
& \left.\varphi_{\epsilon}^{(n)}\left(x_{0}, v, t\right)\right|_{v_{1}<0}=\Theta_{\epsilon, A}^{(n)}\left(x_{0}, t\right)=\Theta_{\epsilon}^{(n-1)}\left(x_{0}, t\right) \\
& \left.\varphi_{\epsilon}^{(n)}(-L, v, t)\right|_{v_{1}>0}=\Theta_{\epsilon, A}^{(n)}(-L, t)=f_{+}(t) \\
& \varphi_{\epsilon}^{(n)}(x, v, 0)=\Theta_{\epsilon, A}^{(n)}(x, 0)=g(x)
\end{aligned}
$$

From Lemma 4.1 we have

$$
\left|\varphi_{\epsilon}^{(n)}(x, v, t)-\Theta_{\epsilon, A}^{(n)}(x, t)\right| \leq \epsilon C \forall x \in D_{1}, v \in S, t \in[0, T]
$$

where the constant $C$ is independent of $n, \epsilon, x, v$ and $t$. Using (4.3) and the coupling conditions we have $\forall t \in[0, T]$

$$
\begin{aligned}
& \left|\Theta_{\epsilon}^{(n)}\left(-x_{0}, t\right)-\Theta_{\epsilon, A}^{(n)}\left(-x_{0}, t\right)\right| \\
& =\mid \frac{\int_{v_{1}>0} v_{1} \varphi_{\epsilon}^{(n)}\left(-x_{0}, v, t\right) d v \quad \int_{v_{1}>0} v_{1} \Theta_{\epsilon, A}^{(n)}\left(-x_{0}, t\right) d v \mid}{\int_{v_{1}>0} v_{1} d v}-\frac{\int_{v_{1}>0} v_{1} d v}{\leq \sup _{v \in S}\left|\varphi_{\epsilon}^{(n)}\left(-x_{0}, v, t\right)-\Theta_{\epsilon, A}^{(n)}\left(-x_{0}, t\right)\right| \leq \epsilon C}
\end{aligned}
$$

At the point $x_{0}$ we have by the weak maximum principle for parabolic equations

$$
\left|\Theta_{\epsilon}^{(n)}\left(x_{0}, t\right)-\Theta_{G}\left(x_{0}, t\right)\right| \leq\left(\sup _{t \in[0, T]}\left|\Theta_{\epsilon}^{(n)}\left(-x_{0}, t\right)-\Theta_{G}\left(-x_{0}, t\right)\right|\right)\left|\Theta_{H}^{D_{2}}\left(x_{0}, t\right)\right|
$$

$\Theta_{H}^{D_{2}}$ is the solution of the diffusion equation in $D_{2}$ with the boundary values

$$
\Theta_{H}^{D_{2}}(L, t)=0, \Theta_{H}^{D_{2}}\left(-x_{0}, t\right)=1
$$

and the initial value

$$
\Theta_{H}^{D_{2}}(x, 0)=\frac{-x+L}{x_{0}+L}
$$

$\Theta_{H}^{D_{2}}$ in (4.5) is constant w.r.t. $t, \Theta_{H}^{D_{2}}=\frac{-x+L}{x_{0}+L}$. Trivial but important is

$$
\Theta_{H}^{D_{2}}\left(x_{0}, t\right)=\frac{-x_{0}+L}{x_{0}+L}=k<1
$$


$\left|\Theta_{\epsilon}^{(n)}\left(x_{0}, t\right)-\Theta_{G}\left(x_{0}, t\right)\right| \leq k \sup _{t \in[0, T]}\left|\Theta_{\epsilon}^{(n)}\left(-x_{0}, t\right)-\Theta_{G}\left(-x_{0}, t\right)\right| \quad \forall t \in[0, T]$

At $-x_{0}$ one can prove analogously

$$
\left|\Theta_{\epsilon, A}^{(n+1)}\left(-x_{0}, t\right)-\Theta_{G}\left(-x_{0}, t\right)\right| \leq\left(\sup _{t \in[0, T]}\left|\Theta_{\epsilon, A}^{(n+1)}\left(x_{0}, t\right)-\Theta_{G}\left(x_{0}, t\right)\right|\right)\left|\Theta_{H}^{D_{1}}\left(-x_{0}, t\right)\right|
$$

$\Theta_{H}^{D_{1}}$ is the solution of $(4.2)$ in $D_{1}$ with

$$
\Theta_{H}^{D_{1}}(-L, t)=0, \Theta_{H}^{D_{1}}\left(x_{0}, t\right)=1, \Theta_{H}^{D_{1}}(x, 0)=\frac{x+L}{x_{0}+L} .
$$

This gives for $x_{0} \neq 0$ and $k<1$ as before

$$
\begin{array}{r}
\left|\Theta_{\epsilon, A}^{(n+1)}\left(-x_{0}, t\right)-\Theta_{G}\left(-x_{0}, t\right)\right| \leq k \sup _{t \in[0, T]}\left|\Theta_{\epsilon, A}^{(n+1)}\left(x_{0}, t\right)-\Theta_{G}\left(x_{0}, t\right)\right| \\
\forall t \in[0, T]
\end{array}
$$

With the definition of $\Theta_{\epsilon, A}^{(n+1)}$ and the coupling condition we get

$$
\Theta_{\epsilon, A}^{(n+1)}\left(x_{0}, t\right)=\left.\varphi_{\epsilon}^{(n+1)}\left(x_{0}, v, t\right)\right|_{v_{1}<0}=\Theta_{\epsilon}^{(n)}\left(x_{0}, t\right),
$$

which yields for all $t \in[0, T]$ using (4.7)

$$
\left|\Theta_{\epsilon, A}^{(n+1)}\left(-x_{0}, t\right)-\Theta_{G}\left(-x_{0}, t\right)\right| \leq k \sup _{t \in[0, T]}\left|\Theta_{\epsilon}^{(n)}\left(x_{0}, t\right)-\Theta_{G}\left(x_{0}, t\right)\right| .
$$

Putting all this together one obtains

$$
\begin{aligned}
& \left|\Theta_{G}\left(-x_{0}, t\right)-\Theta_{\epsilon}^{(n+1)}\left(-x_{0}, t\right)\right| \\
& \leq\left|\Theta_{G}\left(-x_{0}, t\right)-\Theta_{\epsilon, A}^{(n+1)}\left(-x_{0}, t\right)\right|+\left|\Theta_{\epsilon, A}^{(n+1)}\left(-x_{0}, t\right)-\Theta_{\epsilon}^{(n+1)}\left(-x_{0}, t\right)\right| \\
& \leq\left|\Theta_{G}\left(-x_{0}, t\right)-\Theta_{\epsilon, A}^{(n+1)}\left(-x_{0}, t\right)\right|+C \epsilon \quad \operatorname{using}(4.4) \\
& \leq k \sup _{t \in[0, T]}\left|\Theta_{G}\left(x_{0}, t\right)-\Theta_{\epsilon}^{(n)}\left(x_{0}, t\right)\right|+C \epsilon \quad \operatorname{using}(4.9) \\
& \leq k^{2} \sup _{t \in[0, T]}\left|\Theta_{G}\left(-x_{0}, t\right)-\Theta_{\epsilon}^{(n)}\left(-x_{0}, t\right)\right|+C \epsilon \quad \operatorname{using}(4.6)
\end{aligned}
$$

Iterating this we get the desired estimates of the boundary values at $-x_{0}$ :

$$
\begin{aligned}
& \sup _{t \in[0, T]}\left|\Theta_{G}\left(-x_{0}, t\right)-\Theta_{\epsilon}^{(n+1)}\left(-x_{0}, t\right)\right| \\
& \leq k^{2}\left(k^{2} \sup _{t \in[0, T]}\left|\Theta_{G}\left(-x_{0}, t\right)-\Theta_{\epsilon}^{(n-1)}\left(-x_{0}, t\right)\right|+C \epsilon\right)+C \epsilon
\end{aligned}
$$




$$
\begin{aligned}
& +\sum_{i=0}^{n} k^{2 i} \cdot C \cdot \epsilon \\
& \leq C k^{2 n+2}+C \cdot \epsilon \cdot \frac{1}{1-k^{2}}, k<1 \\
& \leq C\left(k^{2 n+2}+\epsilon\right)
\end{aligned}
$$

where $C$ is a generic constant.

The boundary value estimate is then extended to the domain $D_{2}$ :

$$
\left|\Theta^{G}(x, t)-\Theta_{\epsilon}^{(n)}(x, t)\right| \leq\left(\sup _{t \in[0, T]}\left|\Theta^{G}\left(-x_{0}, t\right)-\Theta_{\epsilon}^{(n)}\left(-x_{0}, t\right)\right|\right)\left|\Theta_{H}^{D_{2}}(x)\right|
$$

where $\Theta_{H}^{D_{2}}$ is the function in $D_{2}$ defined above. Moreover the above is smaller than

$$
\begin{aligned}
& \sup _{t \in[0, T]}\left|\Theta^{G}\left(-x_{0}, t\right)-\Theta_{\epsilon}^{(n)}\left(-x_{0}, t\right)\right| \\
\leq & C\left(k^{2 n}+\epsilon\right) \quad \forall t \in[0, T], x \in D_{2} .
\end{aligned}
$$

On $D_{1}$ we have

$$
\begin{aligned}
& \left|\varphi_{\epsilon}^{(n)}(x, v, t)-\Theta_{G}(x, t)\right| \\
& \leq\left|\varphi_{\epsilon}^{(n)}(x, v, t)-\Theta_{\epsilon, A}^{(n)}(x, t)\right|+\left|\Theta_{\epsilon, A}^{(n)}(x, t)-\Theta_{G}(x, t)\right| \\
& \leq\left|\Theta_{\epsilon, A}^{(n)}(x, t)-\Theta_{G}(x, t)\right|+C \epsilon \text { using }(4.3) \\
& \leq\left(\sup _{t \in[0, T]}\left|\Theta_{G}\left(x_{0}, t\right)-\Theta_{\epsilon, A}^{(n)}\left(x_{0}, t\right)\right|\right)\left|\Theta_{H}^{D_{1}}(x)\right|+\epsilon C
\end{aligned}
$$

where $\Theta_{H}^{D_{1}}$ is the function defined above on domain $D_{1}$

$$
\begin{aligned}
& \leq \sup _{t \in[0, T]}\left|\Theta_{G}\left(x_{0}, t\right)-\Theta_{\epsilon, A}^{(n)}\left(x_{0}, t\right)\right|+\epsilon C \\
& =\sup _{t \in[0, T]}\left|\Theta_{G}\left(x_{0}, t\right)-\Theta_{\epsilon}^{(n-1)}\left(x_{0}, t\right)\right|+\epsilon C \quad(4.8), \\
& \leq k \sup _{t \in[0, T]}\left|\Theta_{G}\left(-x_{0}, t\right)-\Theta_{\epsilon}^{(n-1)}\left(-x_{0}, t\right)\right|+\epsilon C \\
& \leq k C\left(k^{2 n-2}+\epsilon\right)+\epsilon C \quad \text { as before } \\
& \leq C k^{2 n}+\epsilon C, \quad \forall x \in D_{1}, \quad \forall v \in S, \forall t \in[0, T],
\end{aligned}
$$

where $C$ is some constant and $k<1$.

The uniform estimates in $x, v$ and $t$ are immediately translated into $\mathcal{L}^{\infty}\left(D_{2}, \mathcal{L}^{2}[0, T]\right)$ respectively $\mathcal{L}^{\infty}\left(D_{1} \times S, \mathcal{L}^{2}[0, T]\right)$ estimates: 
Then

$$
\begin{aligned}
\left\|\varphi_{\epsilon}-\Theta_{G}\right\| & \leq\left\|\varphi_{\epsilon}-\varphi_{\epsilon}^{(n)}\right\|+\left\|\varphi_{\epsilon}^{(n)}-\Theta_{G}\right\| \\
\left\|\Theta_{\epsilon}-\Theta_{G}\right\| & \leq\left\|\Theta_{\epsilon}-\Theta_{\epsilon}^{(n)}\right\|+\left\|\Theta_{\epsilon}^{(n)}-\Theta_{G}\right\|
\end{aligned}
$$

Using Theorem 3.7, in particular the geometric convergence, and the above we get:

$$
\left\|\varphi_{\epsilon}-\Theta_{G}\right\| \text { and }\left\|\Theta_{\epsilon}-\Theta_{G}\right\|
$$

are smaller than

$$
C_{\epsilon}\left(\sigma_{\epsilon}\right)^{n}+C\left(k^{2 n}+\epsilon\right)
$$

for any $n$, where $k$ and $\sigma_{\epsilon}$ are smaller than 1 for all $\epsilon$. Therefore $\left\|\varphi_{\epsilon}-\Theta_{G}\right\|$ as well as $\left\|\Theta_{\epsilon}-\Theta_{G}\right\|$ are for $\epsilon$ fixed smaller than

$$
\lim _{n \rightarrow \infty}\left[C_{\epsilon}\left(\sigma_{\epsilon}\right)^{n}+C\left(k^{2 n}+\epsilon\right)\right]=C \cdot \epsilon .
$$

This gives the convergence result for $\epsilon \rightarrow 0$ and also the estimate in the theorem.

\section{$5 \quad$ Numerical Results}

We calculate an example for the equations in Section 4 with different parameters $\epsilon$.

The dimension $N$ is here equal to 1 , the operator $K$ is defined by $K \varphi=\frac{1}{2} \int_{-1}^{1} \varphi(v) d v$. The constant $D$ in the diffusion approximation is then $\frac{1}{3}$. The overlapping domains are $\left[-L, x_{0}\right]=[-1,0.02]$ and $\left[-x_{0}, L\right]=[-0.02,1]$. The boundary conditions are $f_{+}=1$ and $f_{-}=2$.

In the following figures the macroscopic quantities, i.e. $\frac{1}{2} \int_{-1}^{1} \varphi(x, v, t) d v$ in $D_{1}$ and $\Theta(x, t)$ in $D_{2}$ are plotted. The figures show the coupled solutions for overlapping and nonoverlapping domains. $\epsilon$ in the kinetic equation in $D_{1}$ is equal to 0.1 resp. 0.025 resp. 0.005 . The reference solution is the kinetic solution in the whole domain with $\epsilon=0.1$ resp. 0.025 resp. 0.005 in $[-1,0] . \epsilon$ in $[0,1]$ is equal to 0.005 . This is nearly the aerodynamic regime. In the last figure the kinetic reference solution is the same as the aerodynamic solution in the whole domain.

In all calculations the solution is shown at a time $t=T$ so large that a stationary state has been reached. 
large, but in contrast the results for $\epsilon_{1}$ small become worse. So to obtain better solutions one should use a variable overlapping parameter $x_{0}$ depending on the relation of the mean free paths in the two domains.

We remark that in situations with strongly contrasted Knudsen numbers not near a local equilibrium the results may differ much more. In these cases a more exact analysis is required to get the appropriate coupling conditions. This will be considered in a further publication.

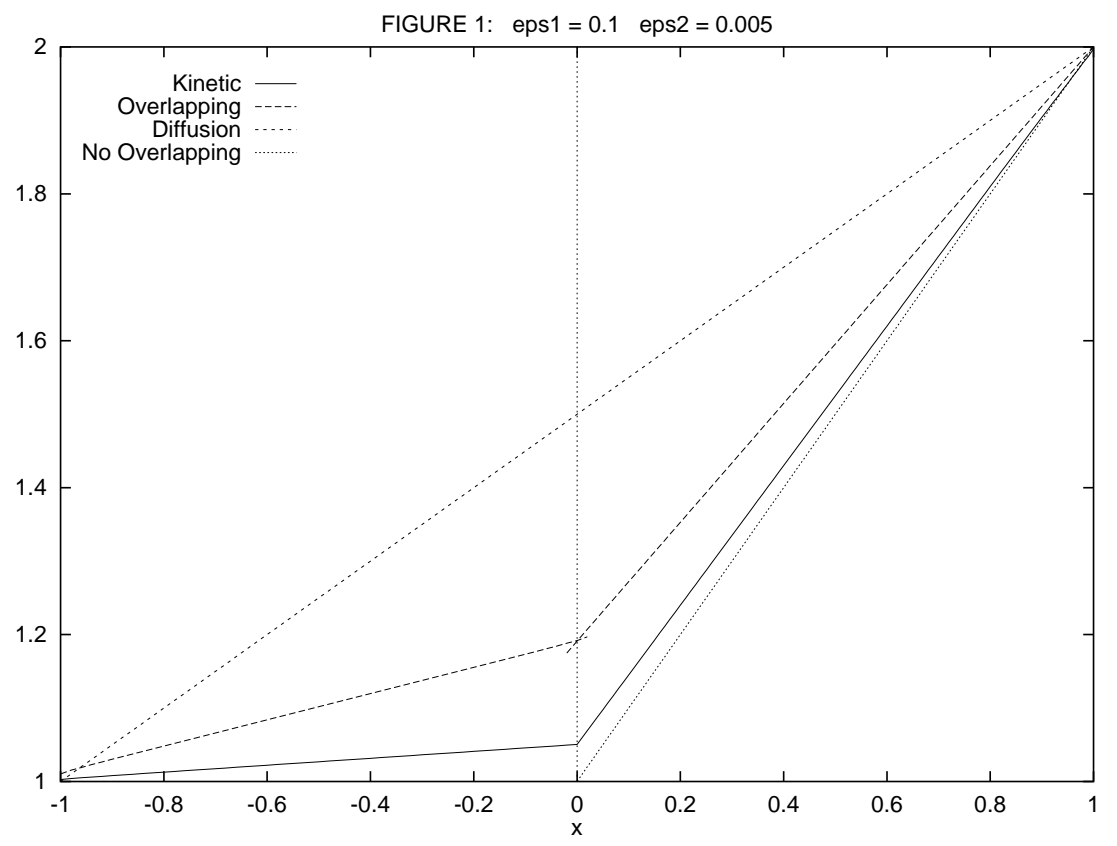



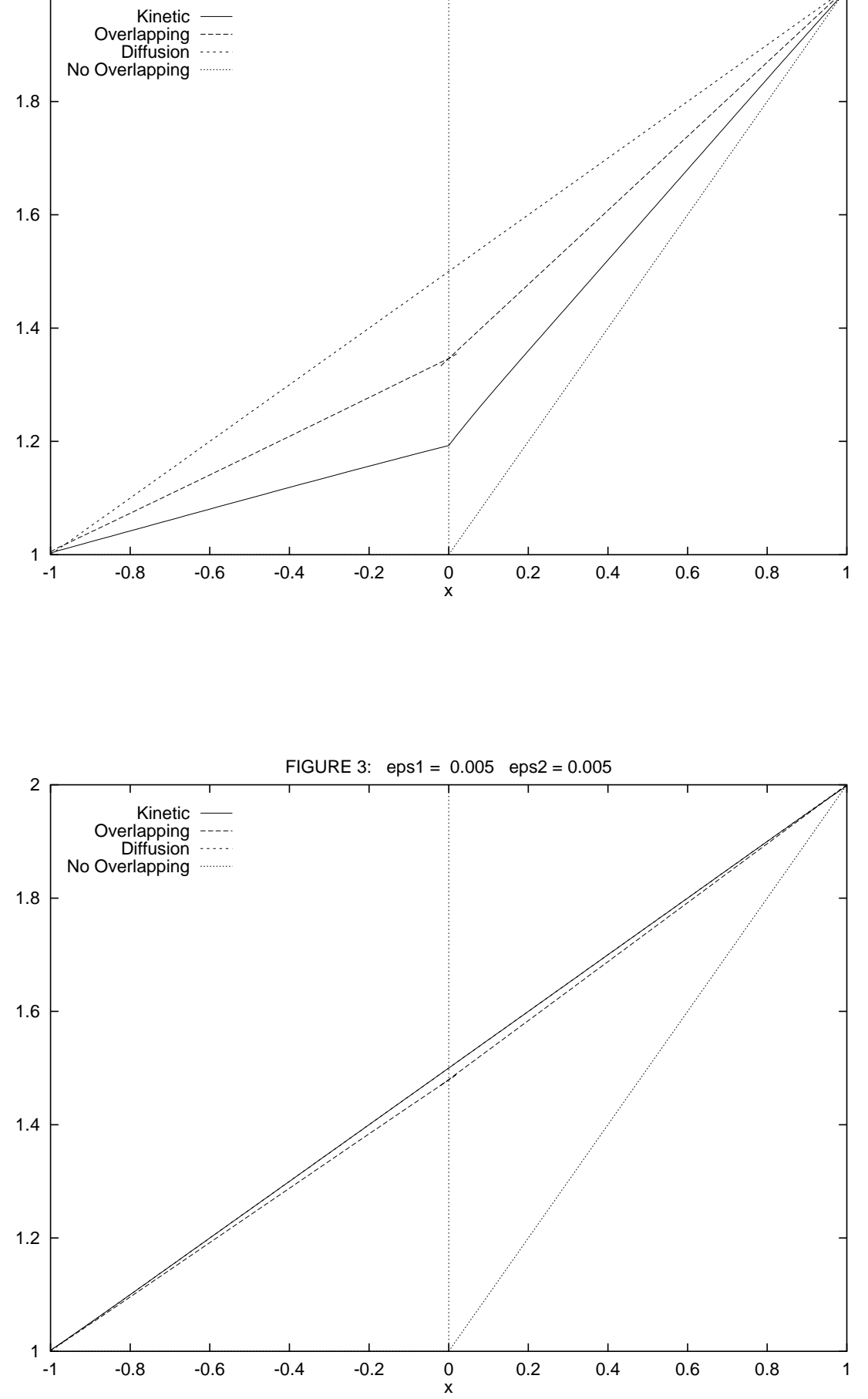
Giering for valuable remarks and discussions.

\section{References}

[1] C. Bardos, R. Santos, R. Sentis, Diffusion Approximation and Computation of the Critical Size, TAMS, Vol. 284, No. 2, p. 617, 1984

[2] A. Bensoussan, J.L. Lions, G.C. Papanicolaou, Boundary Layers and Homogenization of Transport Processes, Publ. RIMS, Kyoto Univ. 15, p. 52, 1979

[3] J.F. Bourgat, P. Le Tallec, D. Tidriri, Y. Qiu, Numerical Coupling of Nonconservative or Kinetic Models with the Conservative Compressible Navier-Stokes Equations, INRIA preprint No. 1426, 1992

[4] R. Dautray, I.L. Lions, Mathematical Analysis and Numerical Methods for Science and Technology, Volume 2, Springer 1988

[5] F. Golse, Applications of the Boltzmann Equation within the Context of Upper Atmosphere Vehicle Aerodynamics, Computer Meth. in Engineer. and Appl. Mech. Vol. 75 , p.299, 1985

[6] H. Grad, Asymptotic Theory of the Boltzmann Equation II, Rarefied gas dynamics, Proceedings of the 3. International symposium 1962, ed. J.A. Laurman, Academic Press, p. 26, 1963

[7] R. Illner, H. Neunzert, Domain Decomposition: Linking Kinetic and Aerodynamic Description, AGTM preprint No. 90, Kaiserslautern, 1993

[8] A. Klar, Domain Decomposition for Kinetic and Aerodynamic Equations, PhD Thesis, Kaiserslautern, 1994

[9] H.O. Kreiss, J. Lorenz, Initial-Boundary Value Problems and the Navier-Stokes Equations, Academic Press 1989

[10] P.L. Lions, On the Schwarz Alternating Method I, II in First and Second International Symposium on Domain Decomposition methods for P.D.E., SIAM, Philadelphia 1988 and 1989

[11] A. Lukschin, H. Neunzert, J. Struckmeier, Interim Report for the Hermes Project DPH 6174/91, 1992

[12] H.A. Schwarz, Über einige Abbildungsaufgaben, Ges. Math. Abh., p. 65, 1869 